\title{
Structural Health Monitoring of Tall Buildings with Numerical Integrator and Convex-Concave Hull Classification
}

\author{
Suresh Thenozhi, ${ }^{1}$ Wen Yu, ${ }^{1}$ \\ Asdrúbal López Chau, ${ }^{2}$ and Xiaoou $\mathrm{Li}^{2}$ \\ ${ }^{1}$ Departamento de Control Automatico, CINVESTAV-IPN, 07360 México, DF, Mexico \\ ${ }^{2}$ Departamento de Computacion, CINVESTAV-IPN, 07360 México, DF, Mexico
}

Correspondence should be addressed to Wen Yu, yuw@ctrl.cinvestav.mx

Received 1 September 2012; Accepted 15 November 2012

Academic Editor: Huaguang Zhang

Copyright (C) 2012 Suresh Thenozhi et al. This is an open access article distributed under the Creative Commons Attribution License, which permits unrestricted use, distribution, and reproduction in any medium, provided the original work is properly cited.

An important objective of health monitoring systems for tall buildings is to diagnose the state of the building and to evaluate its possible damage. In this paper, we use our prototype to evaluate our data-mining approach for the fault monitoring. The offset cancellation and high-pass filtering techniques are combined effectively to solve common problems in numerical integration of acceleration signals in real-time applications. The integration accuracy is improved compared with other numerical integrators. Then we introduce a novel method for support vector machine (SVM) classification, called convex-concave hull. We use the Jarvis march method to decide the concave (nonconvex) hull for the inseparable points. Finally the vertices of the convex-concave hull are applied for SVM training.

\section{Introduction}

Structural vibration control and structural health monitoring (SHM) technologies are concerned with the safety of building structures. The original problem in SHM is to find the structural damage and its location by performing some statistical pattern recognition on the measured data [1] termed as feature extraction. The damage caused by environmental loads should be repaired; otherwise it will grow with time and may lead to total system failure. Dynamic parameters like acceleration, velocity, and displacement play an important role in determining the structure dynamics [2]. Especially in the case of bridges, displacement is a vital information [3]. Traditional displacement sensors are difficult to install on bridges and cannot be useful especially during a seismic activity. Another popular sensing method is 
the global positioning system (GPS). But its usage is affected by bad weather, electromagnetic noise, satellite cycling, and cost. Laser Doppler vibrometer (LDV) is another option, which is limited due to its installation scheme and cost [4]. Because the structure of the accelerometer is very simple and it does not need any relative reference position, most of acceleration and tilt measurements use accelerometers [5]. Wireless networks (WNs) have been used to avoid the high cost of traditional generic wired systems [6].

Since the measured acceleration signal from an accelerometer contains offset and lowfrequency noise, it is not convenient to integrate the acceleration signal directly. The offset and unknown initial conditions of an accelerometer cause drifts during integration. A driftfree numerical integrator was proposed in [7]. The drift elimination method in the frequency domain was designed in [8]. The main problem of these filter-like numerical integrators is that they have to use large time constants to avoid the drift. Baseline correction is an alternative method to avoid drift during integration [5]. The main problem of the baseline correction integrator is that the low-frequency noise was removed using a window filter designed for a particular input signal, so they cannot be applied for online estimation. Some SHM use observers to estimate velocity and position using the measured acceleration signal $[9,10]$. However, the behavior of a filter with a large time constant is far from an ideal integrator.

The structural health monitoring of tall buildings usually uses vibration data. The damage reflects the changes of structural parameters, such as the stiffness and damping coefficients. Only few research used a data-mining technique on SHM. In [11] classification methods were used to determinate the modal parameters, such as the structure's natural frequencies, the vibration intensity, and the damping coefficients.

Support vector machine (SVM) is a highly desirable classification method, because it offers a hyperplane that represents the largest separation (or margin) between the two classes [12]. However, it needs to solve the quadratic programming (QP) in order to find a separation hyperplane, which causes an intensive computational complexity. A method of reducing training data is to use the geometric properties of SVM [13]. Convex hull has been applied in training SVM [14]. In computational geometry, a number of algorithms are known for computing the convex hull for a finite set of points. The Graham scan [15] finds all vertices of the convex hull ordered along its boundary by computing the direction of the cross-product of the two vectors. The Jarvis march (gift wrapping) [16] identifies the convex hull by angle comparisons and wrapping a string around the point set. The Divide and Conquer method [17] is applicable to the three-dimensional case. The incremental convex hull [18] and quick hull [19] algorithms consist of eliminating some points so that the problems are easily solved. By using a nonconvex loss function, it forms a nonconvex SVM. But some good properties of SVM, for example, the maximum margin, cannot be guaranteed [20], because the intersection parts of data sets are not satisfied convex conditions.

In this paper, a baseline correction scheme is applied to the output of a filter-like numerical integrator. Instead of using a single baseline correction [5] may not guarantee a drift-free integration. By using multiple baseline correction, the time constant of the proposed low-pass filter can be reduced to a smaller value compared to the integrator in [7].

The data-mining technique in this paper does not identify structure parameters but compares the changes from the model output and the real output. Since the model of the building does not change, the vibrations compared with the model can be used to diagnose faults in the building.

We also propose a new algorithm to search the border points, called convex-concave hull. By using the Jarvis march method [16], we first find the convex hulls of the data 
set. Then concave hulls are formed using the vertices of the convex hull. In this way, the misleading points in the intersection become the vertices of the concave hulls. The classification accuracy is increased a lot compared with the above papers whereas training time is decreased considerably.

Finally, we design a shake bed and a five-level structure to test our methods. The real experiment results give validation of our methods.

\section{Numerical Integrator for Accelerometer Measurements}

An accelerometer can be regarded as a single-degree-of-freedom (SDOF) mechanical system [21]. It can be modeled using a simple mass $m$ often called as the proof-mass, attached to a spring of stiffness $k$ and a dashpot with damping coefficient $c$ that is attached to a base

$$
m \ddot{x}_{r}(t)+c \dot{x}_{r}(t)+k x_{r}(t)=-m \ddot{x}(t) .
$$

Here $\ddot{x}(t)$ is the acceleration acting on the accelerometer and $\ddot{x}_{r}(t)$ is the relative acceleration of the proof-mass with respect to the base. Other than the acceleration, the accelerometer output signal $a(t)$ contains offset and noise. An accelerometer has a bias termed as offset voltage or $0 g$-offset measured under the absence of motion or gravity $(0 g)$, which is normally equal to the half of its power supply $\left(V_{d d} / 2\right)$. This offset may vary from one sensor to another. The main causes for the offset variation are the sensing material, temperature changes, supply voltage deviation, mechanical stress, and trim errors [22]. This change in the offset from its ideal value is termed as the offset error. The knowledge of this offset error will help to remove the bias from the acceleration signal effectively. The accelerometer output signal can be represented as $[21,23]$

$$
a(t)=k_{a} \ddot{x}(t)+w(t)+d,
$$

where $w(t)$ is the noise and disturbance effects on the measurement and $d$ denotes the $0 g$ offset. Mathematically the velocity $\dot{x}(t)$ and position $x(t)$ are calculated by integrating the acceleration $\ddot{x}(t)$ :

$$
\dot{x}(t)=\int_{0}^{t} \ddot{x}(\tau) d \tau+\dot{x}(0), \quad x(t)=\int_{0}^{t} \int_{0}^{\tau} \ddot{x}(\tau) d \tau d t+\dot{x}(0) t+x(0)
$$

where $\dot{x}(0)$ and $x(0)$ are the initial velocity and position, respectively.

Aliasing is unavoidable when digitizing the analog signals using ADC with a constant sampling frequency. It has been shown that the aliasing can cause low-frequency errors in the acceleration signal [24]. During the analog-to-digital conversion the frequency components above the Nyquist rate are folded back into the bandwidth of interest. Thus the accelerometer output signal in (2.2) can be modified as

$$
a(t)=k_{a} \ddot{x}(t)+\ddot{x}_{s}(t)+w(t)+d,
$$




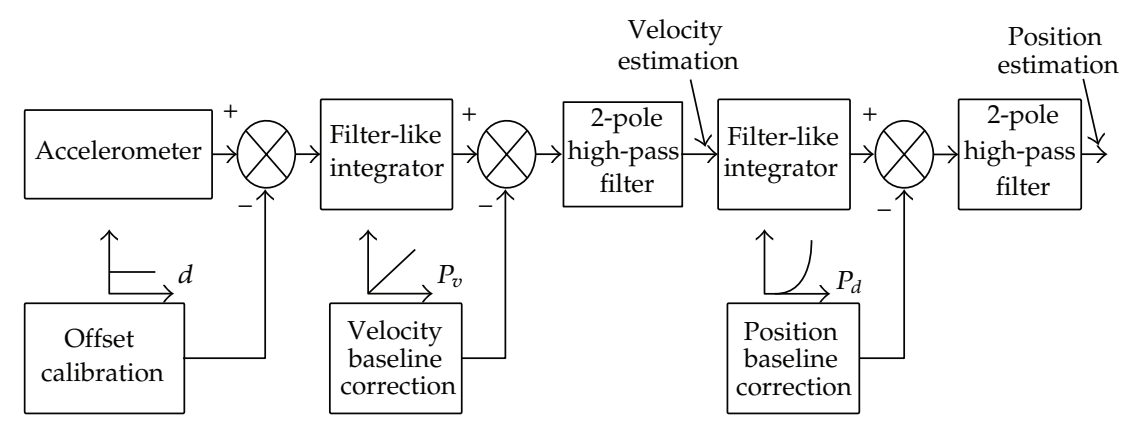

Figure 1: Scheme of the proposed numerical integrator.

where $\ddot{x}_{s}(t)$ is the aliasing content due to sampling. This low-frequency content will be amplified during the integration process. This error can be minimized by using an anti-aliasing filter between the accelerometer and the data acquisition card. The ADC sampling rate needs to be high enough compared to this filter cut-off frequency and the sampling should be done in uniform time intervals.

It is impossible to eliminate the noise and keep the acceleration signal feature at the same time. The aim is to minimize the estimation error caused by $\ddot{x}_{s}(t), w(t)$, and $d$. Most of the accelerometers come with antialiasing filters, which minimizes the aliasing component $\ddot{x}_{s}(t)$. The acceleration gain $k_{a}$ can be easily eliminated by performing calibration. In order to overcome the above problems, the proposed method combines the baseline correction and the filter-like integrator effectively. The scheme of the proposed numerical integrator for the accelerometer measurements is shown in Figure 1. The methods for minimizing the effects of $w(t)$ and $d$ on estimation are discussed below.

Using multiple baseline corrections, a low-pass filter with a small time constant can be achieved to approximate the ideal integrator behavior. The transfer function of the filter-like integrator is

$$
G(s)=\frac{\alpha}{s+\beta}
$$

where $\alpha$ is the gain and $\beta$ is a time constant, which is close to zero. When $\beta$ is zero, the above system reduces to an ideal integrator.

The ideal integrator $(\beta=0)$ amplifies the DC offset quickly. On the other hand, a large $\beta$ reduces the phase accuracy. Selecting an optimal $\beta$ is a trade-off problem between a minimal DC offset and a better phase accuracy. Usually we select $\alpha=1$. $\beta$ is between 0.1 and 0.01 .

As this offset changes with time, this circuit needs frequent calibration, which may be difficult in some cases. Another option is to use a high-pass filter in order to remove the low-frequency DC components. The major drawback of this approach is that the high-pass filter introduces a phase error in the cut-off frequency range. Here it is assumed that the accelerometer is at rest before the seismic activity. In that case, the only output is the $0 g$-offset.

$$
a(t+n)= \begin{cases}d & \text { if } n<0 \\ x(t+n)+d & \text { if } n \geq 0\end{cases}
$$


Finally the offset free acceleration is

$$
\ddot{\widetilde{x}}(t)=r(t)=\ddot{x}(t) .
$$

It is clear that the above algorithm can remove the pure DC component completely. As discussed earlier, there exist other sources of offset other than $0 g$-offset. In that case, the offset Calibration will not be able to eliminate completely the drift from velocity estimation. Now the drifted baseline $p_{v}(t)$ needs to be calculated, which can be represented in a polynomial form as

$$
p_{v}(t)=p_{v 2} t^{2}+p_{v 1} t+p_{v 0}
$$

where $p_{v i}$ are the coefficients to be determined. There are many ways to obtain the coefficients. In this paper, the curve fitting technique using the least-square method (LMS) is used to determine the polynomial coefficients. Once the baseline for the velocity is obtained, it is subtracted from the integrated acceleration signal, to get a drift-free velocity signal. It is found that a second-order polynomial is enough to get a good solution. Increasing the order of the polynomial causes numerical oscillation.

Finally the same methodology is applied to perform the baseline correction for the position estimation. It is found that a third-order polynomial gives a good solution. The position baseline $p_{d}(t)$ is designed as

$$
p_{d}(t)=p_{d 3} t^{3}+p_{d 2} t^{2}+p_{d 1} t+p_{d 0}
$$

In order to remove the low-frequency components in (2.4), a high-pass filter is added after the baseline correction as shown in Figure 1. Here a two-pole high-pass filter is used as in [25]. The transfer function of the single-gain Sallen-Key high-pass filter is

$$
H(s)=\frac{s^{2}}{s^{2}+(2 / \tau) s+\left(1 / \tau^{2}\right)}
$$

where $\tau$ is the filter time constant. The cut-off frequency of the filter is $f_{c}=(1 / 2 \pi \tau)$.

\section{Convex-Concave Hull Classification}

\subsection{Building Model}

Since we need to compare the real vibration signals and the output of the building model, we first need to model the tall buildings. In the case of high-rise flexible buildings, strong winds cause sickness or psychological responses like anxiety to the occupants and also may damage the fragile items. When the vibrations of taller buildings due to the high wind exceed a limit of $0.15 \mathrm{~m} / \mathrm{sec}^{2}$, the human may feel uncomfortable [26]. As a result, the main objective of structural control is to reduce the acceleration response of buildings to a comfortable level. A single-degree-of-freedom structure can be modeled using three components: the mass component $m$, the damping component $c$, and the stiffness component $k$. Among these three 
components, the stiffness component $k$ can be modeled as either a linear or a nonlinear, in other words elastic or inelastic, respectively [27]. Usually the mass is considered as a constant. When an external force $f$ is applied to a structure, it produces changes in its displacement $x(t)$, velocity $\dot{x}(t)$, and acceleration $\ddot{x}(t)$. Using Newton's law, the equation of motion of a linear elastic system subjected to an external force can be written as

$$
m \ddot{x}+c \dot{x}+k x=f .
$$

Then the equation of motion of a linear structure with $n$-degree-of-freedom ( $n$-DOF) can be expressed as

$$
M \ddot{x}(t)+C \dot{x}(t)+K x(t)=F,
$$

where $M, C$ and $K \in \mathbb{R}^{n \times n}$, are the mass, damping and stiffness matrices respectively, $\ddot{x}(t), \dot{x}(t)$ and $x(t) \in \mathbb{R}^{n \times 1}$ are the relative acceleration, velocity and displacement vectors respectively and $F \in \mathbb{R}^{n \times 1}$ is the external force vector. The equation of the motion of a nonlinear structure subjected to ground acceleration $\ddot{x}_{g}(t)$ is

$$
m \ddot{x}+c \dot{x}+f_{s}(x, \dot{x})=-m \ddot{x}_{g} .
$$

The nonlinear force $f_{s}(x, \dot{x})$ in (3.3) can be modeled using the Bouc-Wen model

$$
f_{s}(x, \dot{x})=\tilde{\alpha} k x+(1-\tilde{\alpha}) k \tilde{\eta} f_{r}
$$

In the above expression, $f_{r}$ introduces the nonlinearity, which satisfies the following condition:

$$
\dot{f}_{r}=\frac{\tilde{\delta} \dot{x}-\tilde{v}\left(\tilde{\beta}|\dot{x}|\left|f_{r}\right|^{\tilde{n}-1} f_{r}+\tilde{\gamma} \dot{x}\left|f_{r}\right|^{\tilde{n}}\right)}{\tilde{\eta}}
$$

where $f_{r}$ is the nonlinear time-dependent restoring force and $\widetilde{\delta}, \tilde{\beta}, \tilde{\gamma}, \tilde{v}, \tilde{\eta}$, and $\tilde{n}$ are the parameters, which controls the shape of the hysteresis loops and system degradation. The variables $\widetilde{\delta}, \widetilde{\alpha}, \tilde{\eta}$, and $k$ control the initial tangent stiffness [28]. In the case of $n$-DOF structures, the nonlinear model can be modified as

$$
M \ddot{x}(t)+C \dot{x}(t)+F_{S}(x(t), \dot{x}(t))=-M \Lambda \ddot{x}_{g}(t),
$$

where $\Lambda \in \mathbb{R}^{n \times 1}$ denotes the influence of the excitation force. 


\subsection{Faults Diagnosis}

We assume that $n_{a}$ accelerometers are installed in the building. The numerical integration outputs are velocity $\dot{x}_{a} \in R^{n_{a}}$ and displacement $x_{a} \in R^{n_{a}}$. The fault diagnosis signals are

$$
x_{1}=x_{a}-x \in R^{n_{a}}, \quad x_{2}=\dot{x}-\dot{x}_{a} \in R^{n_{a}} .
$$

We define that a damage does not occur when

$$
a_{1 i} \leq\left|x_{1 i}\right| \leq b_{1 i}, \quad c_{2 i} \leq\left|x_{2 i}\right| \leq d_{2 i}, \quad x_{1}=\left[x_{11} \cdots x_{1 n_{a}}\right]^{T}, \quad x_{2}=\left[x_{21} \cdots x_{2 n_{a}}\right]^{T},
$$

where $i=1 \ldots n_{a}$ and $a_{1 i}, b_{1 i}, c_{2 i}$, and $d_{2 i}$ are previous defined constants. When no damage occurs, we define that the property of $\left(x_{1 i}, x_{2 i}\right)$ is +1 . Otherwise the property of $\left(x_{1 i}, x_{2 i}\right)$ is -1 . In Figure 4, they are marked "o" and "+".

Because the accelerometers work on-line, the sampled data are huge. Normal classification techniques do not work. We use the convex-concave hull method.

\subsection{Convex-Concave Hull}

In many real applications of SVM, the data are not perfectly separated, and the kernel methods are not so powerful for nonlinear separation. The closest points in the convex hulls are no longer support vectors. In this case, the soft margin optimization of SVM can be applied directly to the inseparable sets of the convex hulls. The penal parameter affects the optimal performance of SVM. The optimization becomes a tradeoff between a large margin and a small error penalty.

For the intersection parts, the convex hulls have to be reduced such that the inseparable case becomes separable case [29]. The key disadvantage of this reduced convex hull is that the convex hull has to be calculated in each reducing step [14].

In this paper, we first find the convex hulls of the data set. Then we propose a new algorithm to search the border points; the nonconvex hulls are formed by the vertices of the convex hull. In this way, the misleading points in the intersection become the vertices of the concave hulls. The classification accuracy increases a lot compared with the other convex hull methods whereas training time is decreased considerably.

In order to define the vertices of a convex-concave hull, we used the border points definition $B(X)$, which are the outer border points located on the boundaries of the set $X$. The vertices of a convex-concave hull are the border points' $B(X)$ :

$$
B(X)=V_{\mathcal{C} \mathfrak{H}(X)} \cup V_{\mathcal{N C H}(X)},
$$

where $V_{\mathcal{C A}(\mathrm{X})}$ is the set of vertices of a convex hull and $V_{\mathcal{N C H}(\mathrm{X})}$ is the set of the vertices of a nonconvex hull (concave hull); see Figure 2. A set $S \in R^{n}$ is said to be convex if

$$
\text { if } x_{1}, x_{2} \in S, \quad \text { then } \alpha x_{1}+(1-\alpha) x_{2} \in S, \quad \forall \alpha \in(0,1) \text {. }
$$

The border points $\mathcal{B}(X)$ have the following characteristics. For any two extreme points in $\mathcal{C} \mathcal{A}(X)$, all other points in $X$ are located on one side of the line connecting these two points. 


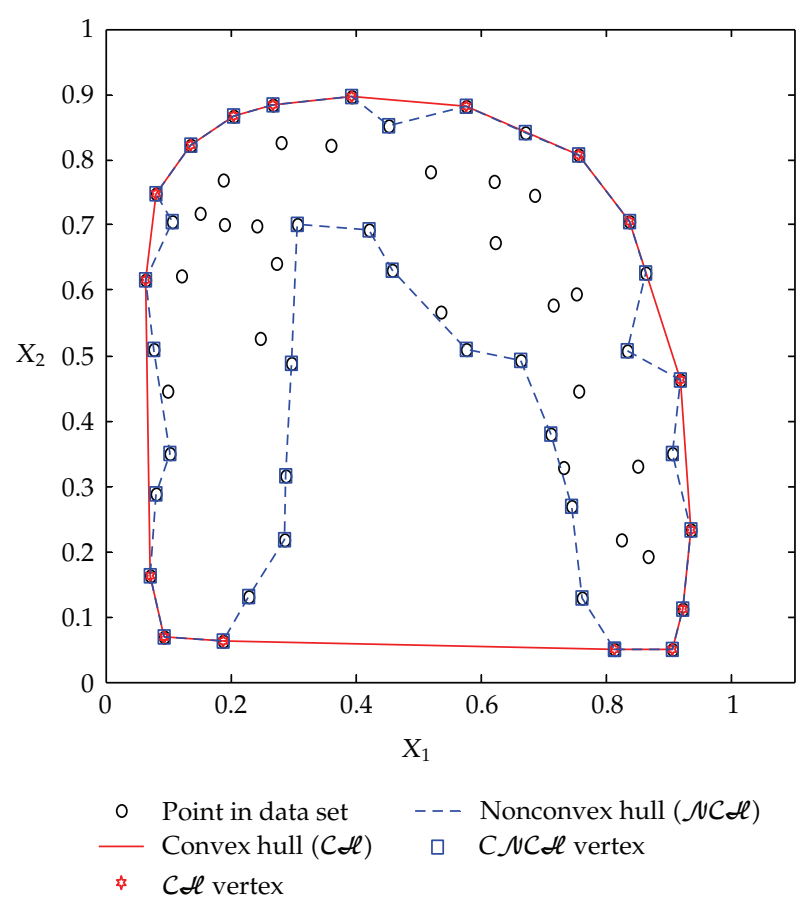

Figure 2: The convex hull and a non convex hull of a set of points.

We use two adjacent extreme points as reference points to detect the concave hull $\mathcal{N C} \mathscr{\ell}(X)$ between them. The convex-concave hull searching scheme behaves good if the data set $X$ has a uniform distribution of elements in a region. However, the distribution of a data set is unknown in advance. Computing the convex-concave hull $B(X)$ directly from $X$ is not appropriate for training the SVM classifier. In order to avoid the density problem, we separate $X$ into $X^{+}$and $X^{-}$and then we create partitions $P^{+}\left(\cup P^{+}=X^{+}\right.$and $\left.\cap P^{+}=\emptyset\right)$ and $P^{-}\left(\cup P^{-}=X^{-}\right.$ and $\cap P^{-}=\emptyset$ ), they will be abbreviated as $P_{i}^{ \pm}$.

The basic idea here is to obtain regions $P_{i}^{ \pm}$where the distribution is more uniform than in the original ones. Convex-concave hull searching is then applied on each $P_{i}^{ \pm}$to reduce the size of $X$ whereas preserving most support vectors. The $V_{\mathcal{C} \mathscr{e}\left(\mathrm{X}^{+}\right)}$and $V_{\mathcal{C} \mathfrak{H}\left(\mathrm{X}^{-}\right)}$are always included in $B\left(X^{ \pm}\right)$regardless whether $X$ is linearly separable or inseparable. In addition, points on the intersection of convex hulls are also included in $B\left(X^{ \pm}\right)$.

The subsets $P_{i}$ can be quickly created by introducing all points in a binary tree of height $h$ and then using the leaves as a version of the original points. Once all points have been introduced in the binary tree, it is possible to look down from height $h_{g}<h$ of the tree and take all leaves as a subset $P_{i}$.

We propose a grid method to preprocess the data set. Figure 3 shows the data set before process.

There are basically two cases for the two-class classification using SVM: the linearly separable and the linearly inseparable. In the nonlinear case, suitable kernel can be applied to map the nonlinear classification into the linear classification. 


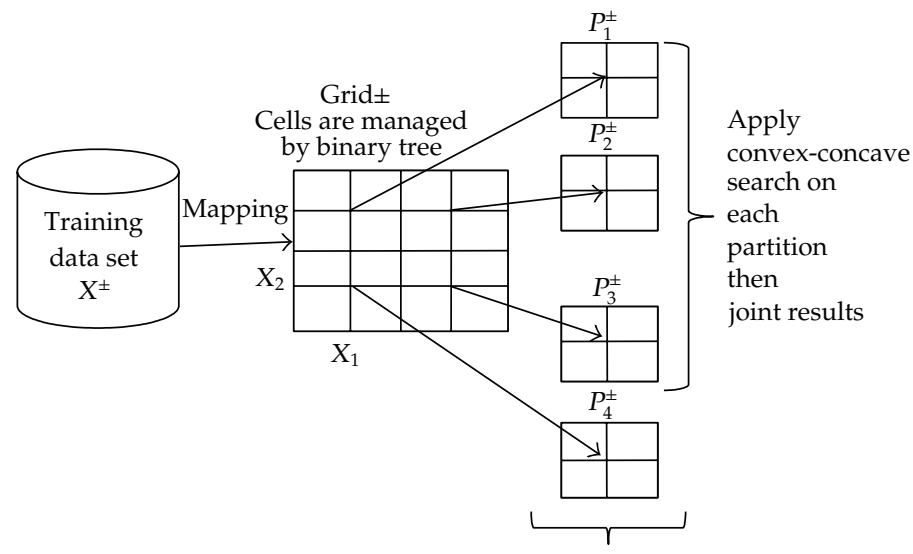

$P_{i}^{ \pm}$: partitions of $X^{ \pm}$,

disjoint groups of adjacent cells of $\mathrm{G}$

Figure 3: Data set preprocessing

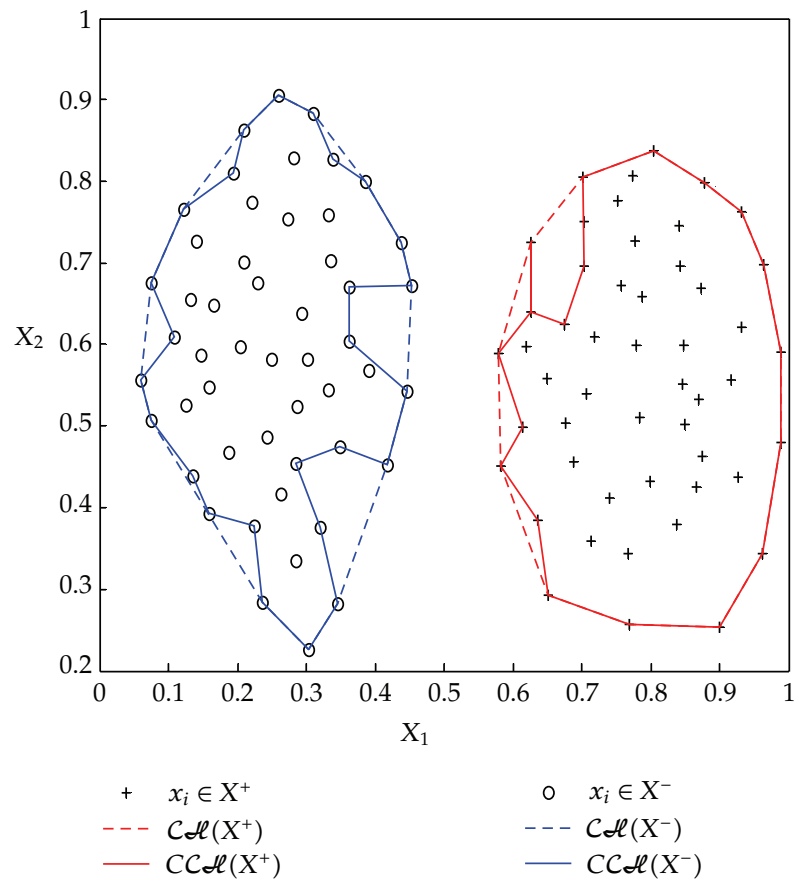

Figure 4: Linearly separable case; $h_{g}=0$.

In the separable case, $\mathcal{C} \mathscr{\ell}\left(X^{+}\right) \cap \mathcal{C} \mathscr{H}\left(X^{+}\right)=\emptyset$, where $X^{ \pm}$represents the elements in $X$ with label +1 or -1 , respectively. It has been demonstrated that if the data set is a linearly separable one, then the SV corresponds to the closest vertices of $\mathcal{C} \mathscr{d}\left(X^{+}\right)$and $\mathcal{C} \mathscr{d}\left(X^{-}\right)$. 


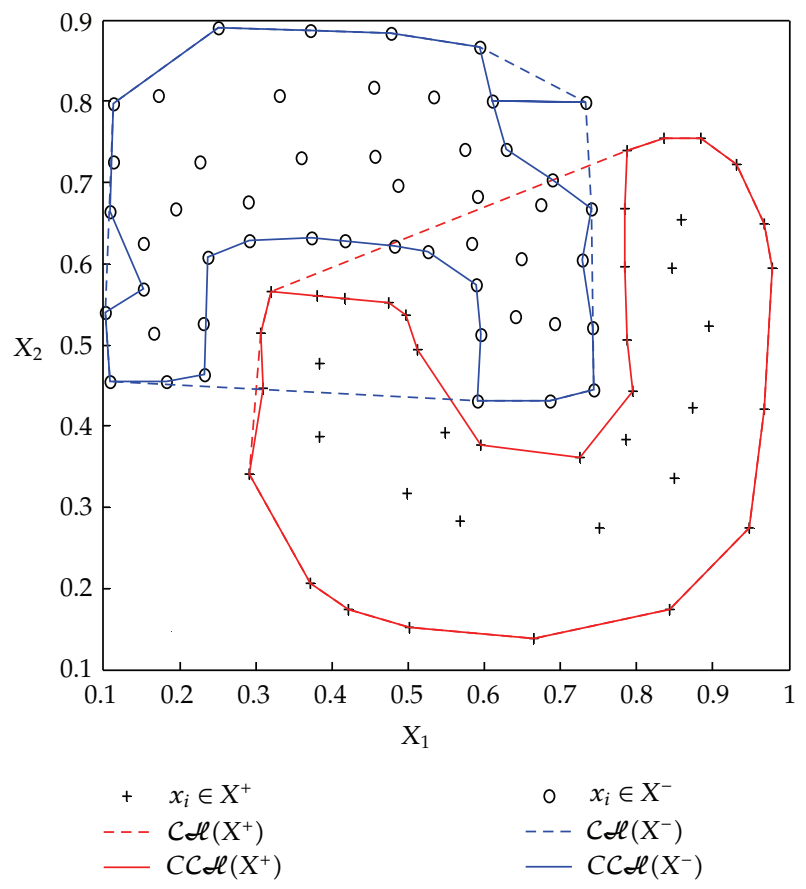

Figure 5: Linearly inseparable case, $h_{g}=0$.

In the linear inseparable case, the convex-hulls-intersect. The convex-hull-based methods do not work well for SVM, because SVs are generally located on the exterior boundaries of data distribution; see Figure 5. Further, the reduced convex hull distorts the original data distribution and produces poor classification accuracy.

After we obtain the vertices of the convex-concave hull, $B(X)=V_{\mathcal{C H}(X)} \cup V_{\operatorname{NCH}(X)}$, these points are sent to train the SVM classifier, that is, to find an optimal hyperplane or to solve the following quadratic programming problem (primal problem):

$$
\begin{aligned}
& \min _{w, b} J(w)=\frac{1}{2} w^{T} w+C \sum_{k=1}^{n} \xi_{k} \\
& \text { subject : } \quad y_{k}\left[w^{T} \varphi\left(x_{k}\right)+b\right] \geq 1-\xi_{k}
\end{aligned}
$$

where $\xi_{k}$ is slack variables to tolerate misclassifications $\xi_{k}>0, k=1 \cdots n, c>0, w_{k}$ is the distance from $x_{k}$ to the hyperplane $\left[w^{T} \varphi\left(x_{k}\right)+b\right]=0$, and $\varphi\left(x_{k}\right)$ is a nonlinear function.

In our convex-concave hull SVM classification, the penal factor $C$ can be selected very small, because all the misleading points almost disappear by the concave algorithm. The classification accuracy is improved. 
The kernel which satisfies the Mercer condition [30] is $K\left(x_{k}, x_{i}\right)=\varphi\left(x_{k}\right)^{T} \varphi\left(x_{i}\right)$. Equation (3.11) is equivalent to the following quadratic programming problem which is a dual problem with the Lagrangian multipliers $\alpha_{k} \geq 0$ :

$$
\begin{aligned}
& \max _{\alpha} J(\alpha)=-\frac{1}{2} \sum_{k, j=1}^{n} y_{k} y_{j} K\left(x_{k}, x_{j}\right) \alpha_{k} \alpha_{j}+\sum_{k=1}^{n} \alpha_{k}, \\
& \text { subject: } \quad \sum_{k=1}^{n} \alpha_{k} y_{k}=0, \quad 0 \leq \alpha_{k} \leq c .
\end{aligned}
$$

Many solutions of (3.12) are zero; that is, $\alpha_{k}=0$, so the solution vector is sparse; the sum is taken only over the nonzero $\alpha_{k}$. The $x_{i}$ which corresponds to nonzero $\alpha_{i}$ is called a support vector. Let $V$ be the index set of support vectors, then the optimal hyperplane is

$$
\sum_{k \in V} \alpha_{k} y_{k} K\left(x_{k}, x_{j}\right)+b=0
$$

The resulting classifier is

$$
y(x)=\operatorname{sign}\left[\sum_{k \in V} \alpha_{k} y_{k} K\left(x_{k}, x\right)+b\right],
$$

where $b$ is determined by the Kuhn-Tucker conditions. The decision hyperplane

$$
\sum_{k \in V_{1}} y_{k} \alpha_{1, k}^{*} K\left(x_{k}, x\right)+b_{1}^{*}=0
$$

\section{Experiments}

In this paper, the velocity and position estimations are evaluated in a shaking table; see Figure 6. The accelerometer we used is Summit Instruments 13203B, which is mounted on the SDOF mechanical structure. The sensitive axis of the accelerometer is mounted parallel to the ground to measure the structure acceleration. A linear magnetic encoder (LM15) position sensor with a resolution of $50 \mu \mathrm{m}$ is used for verifying the estimated position data. The building structure base is mounted on the shaking table; see Figure 7. We first evaluate the numerical integrator proposed in this paper. We use the earthquake signal of Loma Prieta East-West on October 17, 1989. The position estimation is shown in Figure 8.

It has been seen that the proposed filter-like integrator is able to estimate the velocity and position in a reasonable manner. Still there is some error found between the estimated and the measured position. This error is caused due to the phase error introduced by the high-pass filter, which resulted in a small phase delay. But it is found that the estimation will be reasonable for the structural control and health monitoring applications.

Then we use our convex-concave hull classification (CCHSVM) to diagnose the fault. We compare our algorithms with the other four SVM classification methods: SMO [31], LIBSVM [32], clustering-based SVM (CSVM) [33], and the reduced convex hull SVM 


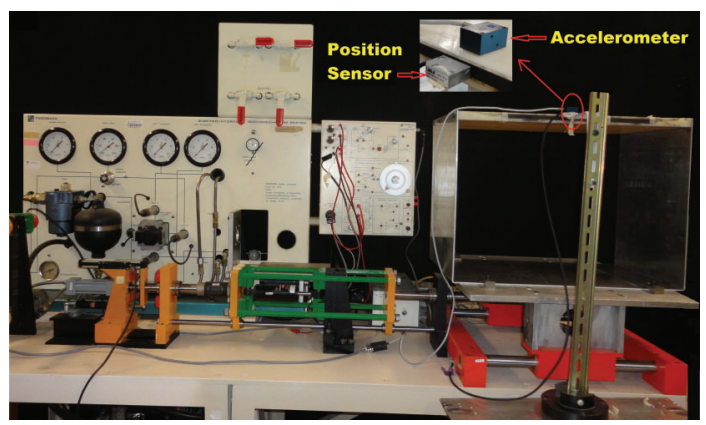

Figure 6: Shaking table.

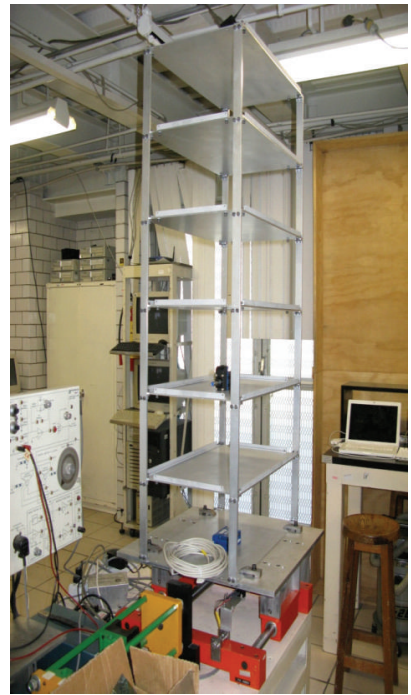

Figure 7: Six-floor structure.

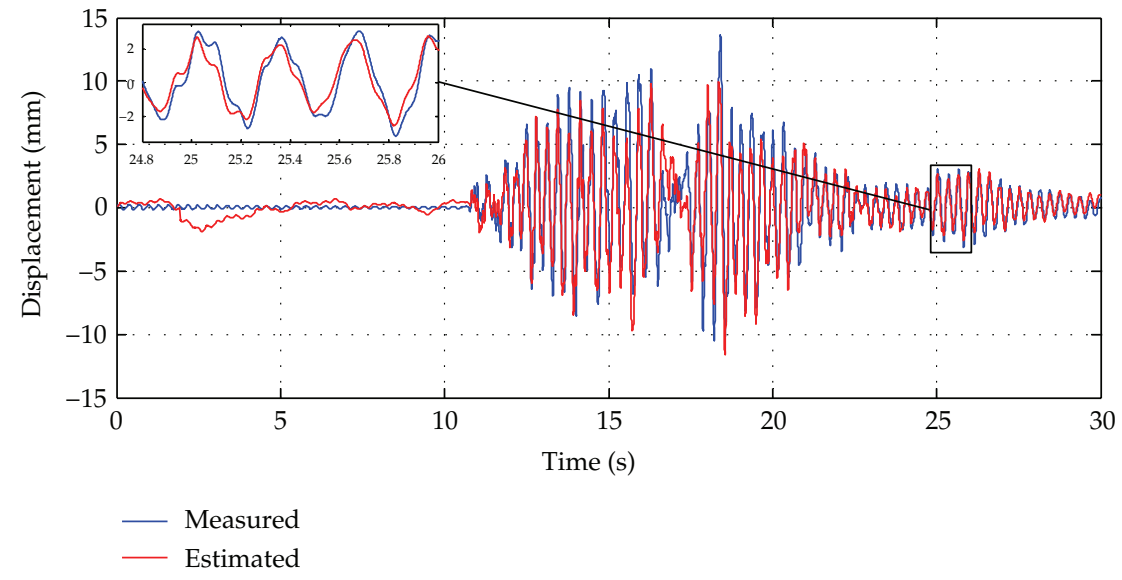

Figure 8: Comparison of the measured and estimated position data. 


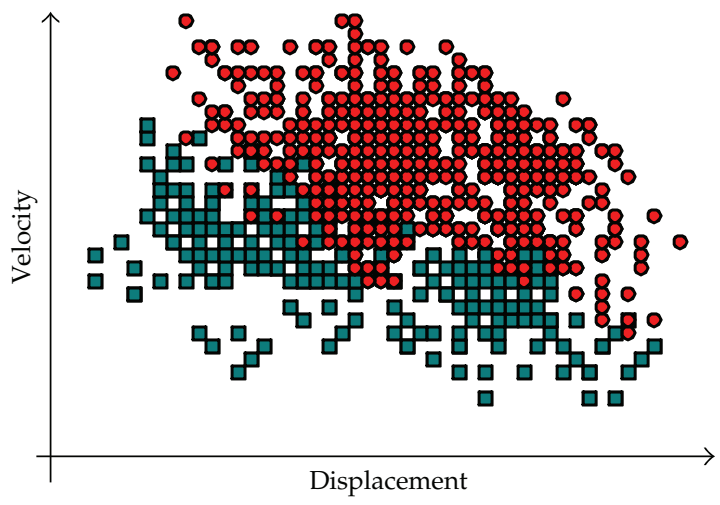

Figure 9: Diagnosis data.

Table 1: Classification results.

\begin{tabular}{lccccccc}
\hline Data set & $h_{g}$ & $K$ & $T_{\text {bp }}$ & $T_{\text {tr }}$ & \#SV & \#BS & Acc \\
\hline $10 K$ & - & - & - & 928 & 171 & - & 99.8 \\
$B(10 K)$ & 7 & 25 & 587 & 241 & 101 & 768 & 99.7 \\
$50 K$ & - & - & - & 12,589 & 1,495 & - & 98.6 \\
$B(50 K)$ & 2 & 15 & 2,635 & 2,887 & 626 & 2,924 & 98.4 \\
$100 K$ & - & - & - & 43,443 & 1,252 & - & 99.8 \\
$B(100 K)$ & 7 & 25 & 3,557 & 3,094 & 770 & 5,319 & 99.4 \\
$200 K$ & - & - & - & 53,643 & 1,173 & - & 99.7 \\
$B(200 K)$ & 9 & 40 & 6,719 & 2,001 & 627 & 4,632 & 98.3 \\
$250 K$ & - & - & - & 69,009 & 6,839 & - & 98.7 \\
$B(250 K)$ & 4 & 15 & 6,501 & 7,181 & 1,237 & 13,171 & 98.4 \\
\hline
\end{tabular}

(RCHSVM) [29]. One part of data is shown in Figure 9. The circles represent the normal case. The squares correspond to a stiffness change in the second floor.

We first examine how does the training data size affect the training time and classification accuracy of our convex-concave hull SVM (CCHSVM). We use $10 K(K=$ $\left.10^{3}\right), 50 K, 100 K, 200 K$, and $250 K$ data set samples to train CCHSVM and SMO. For all data sets, the training data are chosen randomly from $70 \%$ of the data set; the remainig data are used for testing data set. The comparison results are shown in Table 1 . Here $10 \mathrm{~K}$ means to use $10 K$ data to train SMO, $B(10 K)$ means to use $10 K$ data to train CCHSVM. $h_{g}$ and $K$ are CCHSVM algorithm parameters, $T_{\mathrm{bp}}$ is the time to compute border points, $T_{\mathrm{tr}}$ is the training time, \#SV is the number of support vectors, \#BS is the number of vertices of the convexconcave hull, and Acc is the classification accuracy.

We can see that our CCHSVM has less training time than SMO and almost the same classification accuracy with SMO. When the data size is increased, the training time is dramatically increased with SMO, while ours only increases a little. Although the classification accuracy cannot be improved significantly when data size is very large, it does not get worse, and the testing accuracy is still acceptable.

Now we compare our CCHSVM with SMO [31], LIBSVM [32], CSVM [33], and RCHSVM [29] with $40 K$ data set. The comparison results are shown in Table 2. 
Table 2: Classification results.

\begin{tabular}{lccccccc}
\hline Data set & $h_{g}$ & $K$ & $T_{\mathrm{bp}}$ & $T_{\mathrm{tr}}$ & \#SV & \#BS & Acc \\
\hline SMO & - & - & - & 11,243 & 648 & - & 97.3 \\
LIBSVM & - & - & - & 7,130 & 540 & - & 97.8 \\
CSVM & - & - & - & 830 & 310 & - & 97.6 \\
RCHSVM & - & - & - & 19,325 & 350 & - & 90.6 \\
CCHSVM & 4 & 7 & 437 & 548 & 253 & 894 & 96.2 \\
\hline
\end{tabular}

We can see that compared with the other SVM classifiers, our approach has good classification accuracy while the training is significantly faster than other SVM classifiers. The classification accuracy is higher than the other convex hull SVMs.

\section{Conclusions}

In this paper, we use two techniques for structural health monitoring of tall buildings: numerical integrator and convex-concave hull classification (CCHSVM). A numerical integrator is proposed for estimating velocity and position from an acceleration signal. This method combines the multiple baseline corrections and the filtering techniques. The proposed integrator is able to produce stable and accurate estimation.

The CCHSVM uses convex-concave hull and grid before process; the CCHSVM overcomes the problems of slow training of SVM and low accuracy of many geometric properties based SVMs. The key point of our method is that we use the Jarvis march method to decide the concave (nonconvex) hull for the inseparable points.

The experimental results demonstrate that the accuracy of the drift-free integrator is increased by adding the offset cancellation filter, and our approach has good classification accuracy while the training is significantly faster than other SVM classifiers. The classification accuracy is higher than the other convex hull SVMs.

\section{References}

[1] G. W. Housner, L. A. Bergman, T. K. Caughey et al., "Structural control: past, present, and future," Journal of Engineering Mechanics, vol. 123, no. 9, pp. 897-971, 1997.

[2] F. Moschas and S. Stiros, "Measurement of the dynamic displacements and of the modal frequencies of a short-span pedestrian bridge using GPS and an accelerometer," Engineering Structures, vol. 33, no. 1, pp. 10-17, 2011.

[3] K. T. Park, S. H. Kim, H. S. Park, and K. W. Lee, "The determination of bridge displacement using measured acceleration," Engineering Structures, vol. 27, no. 3, pp. 371-378, 2005.

[4] J. J. Lee and M. Shinozuka, "Real-time displacement measurement of a flexible bridge using digital image processing techniques," Experimental Mechanics, vol. 46, no. 1, pp. 105-114, 2006.

[5] D. H. Kim and M. Q. Feng, "Real-time structural health monitoring using a novel fiber-optic accelerometer system," IEEE Sensors Journal, vol. 7, no. 4, pp. 536-544, 2007.

[6] A. Jindal and M. Liu, "Networked computing in wireless sensor networks for structural healthmonitoring," IEEE/Acmtransactions on Networking, vol. 20, no. 4, pp. 1203-1217, 2012.

[7] H. P. Gavin, R. Morales, and K. Reilly, "Drift-free integrators," Review of Scientific Instruments, vol. 69, no. 5, pp. 2171-2175, 1998.

[8] J. G. T. Ribeiro, J. T. P. de Castro, and J. L. F. Freire, "Using the FFT-DDI method to measure displacements with piezoelectric, resistive and ICP accelerometers," in Proceedings of the Conference and Exposition on Structural Dynamics, 2003. 
[9] Y. L. Xu and B. Chen, "Integrated vibration control and health monitoring of building structures using semi-active friction dampers: part I-methodology," Engineering Structures, vol. 30, no. 7, pp. 1789-1801, 2008.

[10] X. Meng, A. H. Dodson, and G. W. Roberts, "Detecting bridge dynamics with GPS and triaxial accelerometers," Engineering Structures, vol. 29, no. 11, pp. 3178-3184, 2007.

[11] E. Forstner and H. Wenzel, "The application of data mining in bridge monitoring projects: exploiting time series data of structural health monitoring," in Proceedings of the 22nd InternationalWorkshop on Database and Expert Systems Applications, 2011.

[12] S. Kalyani, "Classification and assessment of power system security using multiclass SVM," IEEE Transactions on Systems, Man, and Cybernetics C, vol. 41, no. 5, pp. 753-758, 2011.

[13] M. E. Mavroforakis, M. Sdralis, and S. Theodoridis, "A geometric nearest point algorithm for the efficient solution of the SVM classification task," IEEE Transactions on Neural Networks, vol. 18, no. 5, pp. 1545-1549, 2007.

[14] K. P. Bennett and E. J. Bredensteiner, Geometry in Learning, Geometry at Work, Mathematical Association of America, 2000, Edited by C. Gorini.

[15] R. L. Graham, "An efficient algorith for determining the convex hull of a finite planar set," Information Processing Letters, vol. 1, no. 4, pp. 132-133, 1972.

[16] R. A. Jarvis, "On the identification of the convex hull of a finite set of points in the plane," Information Processing Letters, vol. 2, no. 1, pp. 18-21, 1973.

[17] F. P. Preparata and S. J. Hong, "Convex hulls of finite sets of points in two and three dimensions," Communications of the ACM, vol. 20, no. 2, pp. 87-93, 1977.

[18] M. Kallay, "The complexity of incremental convex hull algorithms in Rd," Information Processing Letters, vol. 19, no. 4, p. 197, 1984.

[19] W. Eddy, "A new convex hull algorithm for planar sets," ACM Transactions on Mathematical Software, vol. 3, no. 4, pp. 398-403, 1977.

[20] R. Collobert, F. Sinz, J. Weston, and L. Bottou, "Trading convexity for scalability," in Proceedings of the 23rd International Conference on Machine Learning (ICML '06), pp. 201-208, Pittsburgh, Pa, USA, June 2006.

[21] A. Link and H. J. Von Martens, "Accelerometer identification using shock excitation," Measurement, vol. 35, no. 2, pp. 191-199, 2004.

[22] Freescale Semiconductor, "Accelerometer Terminology Guide," 2007, http://cache.freescale.com/ files/sensors/doc/support_info/SENSORTERMSPG.pdf.

[23] W. H. Zhu and T. Lamarche, "Velocity estimation by using position and acceleration sensors," IEEE Transactions on Industrial Electronics, vol. 54, no. 5, pp. 2706-2715, 2007.

[24] T. S. Edwards, "Effects of aliasing on numerical integration," Mechanical Systems and Signal Processing, vol. 21, no. 1, pp. 165-176, 2007.

[25] R. P. Sallen and E. L. Key, "A practical method of designing RC active filters," IRE Transactions on Circuit Theory, vol. 2, pp. 74-85, 1955.

[26] B. F. Spencer and M. K. Sain, "Controlling buildings: a new frontier in feedback," IEEE Control Systems Magazine, vol. 17, no. 6, pp. 19-35, 1997.

[27] A. C. Nerves and R. Krishnan, "Active control strategies for tall civil structures," in Proceedings of the IEEE 21st International Conference on Industrial Electronics, Control, and Instrumentation, vol. 2, pp. 962-967, November 1995.

[28] R. Garrido and F. J. Rivero-Angeles, "Hysteresis and parameter estimation of MDOF systems by a continuous-time least squares method," Journal of Earthquake Engineering, vol. 10, no. 2, pp. 237-264, 2006.

[29] M. E. Mavroforakis and S. Theodoridis, "A geometric approach to support vector machine (SVM) classification," IEEE Transactions on Neural Networks, vol. 17, no. 3, pp. 671-682, 2006.

[30] N. Cristianini and J. Shawe-Taylor, An Introduction to Support Vector Machines and other Kernel-Based Learning Methods, Cambridge University Press, 2000.

[31] J. Platt, Fast Training of Support Vector Machine using Sequential Minimal Optimization, Advances in Kernel Methods: Support Vector Machine, MIT Press, Cambridge, Mass, USA, 1998.

[32] C.-C. Chang and C.-J. Lin, "LIBSVM: A library for support vector machines," 2001, http://www .csie.ntu.edu.tw/ cjlin/libsvm/.

[33] J. Cervantes, X. Li, W. Yu, and K. Li, "Support vector machine classification for large data sets via minimum enclosing ball clustering," Neurocomputing, vol. 71, no. 4-6, pp. 611-619, 2008. 


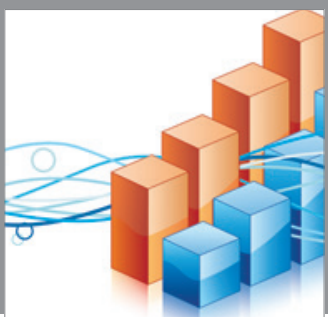

Advances in

Operations Research

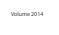

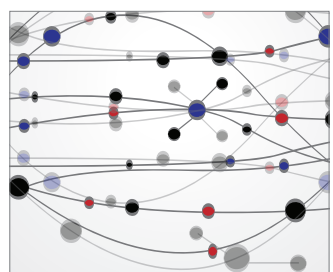

\section{The Scientific} World Journal
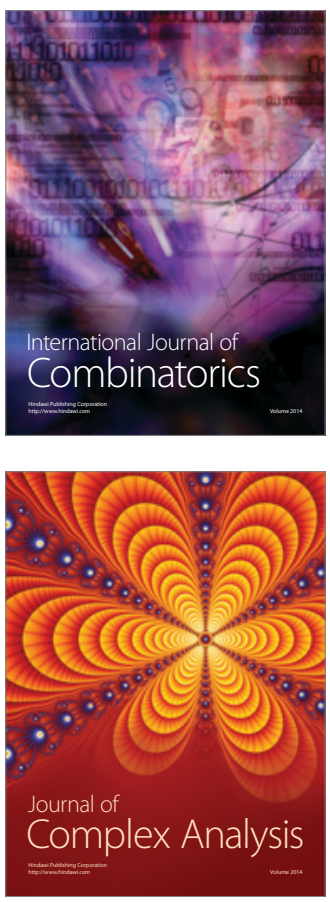

International Journal of

Mathematics and

Mathematical

Sciences
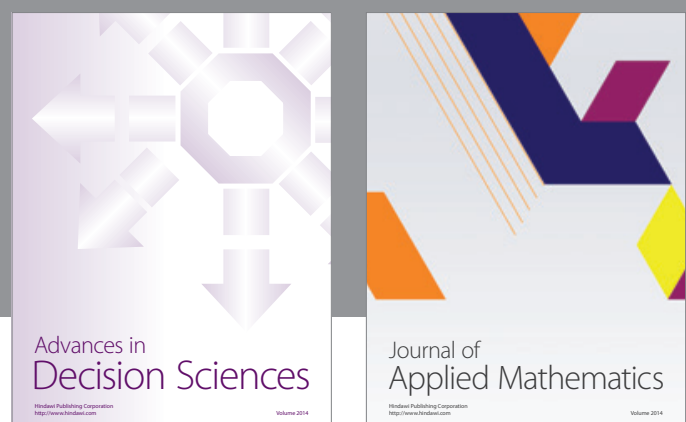

Journal of

Applied Mathematics
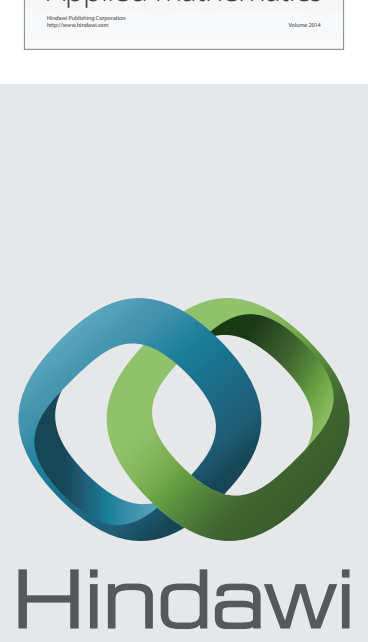

Submit your manuscripts at http://www.hindawi.com
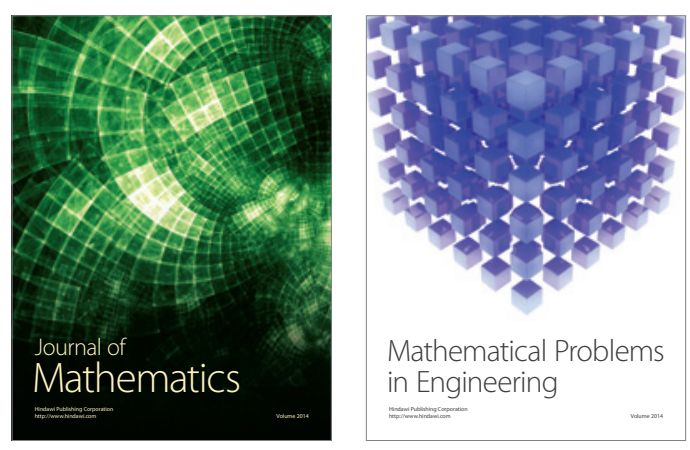

Mathematical Problems in Engineering
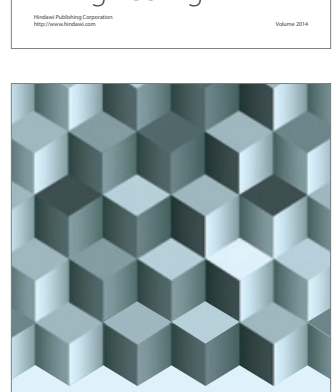

Journal of

Function Spaces
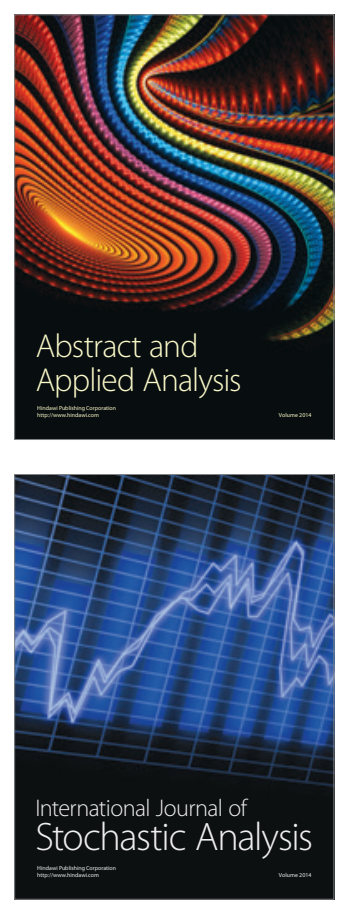

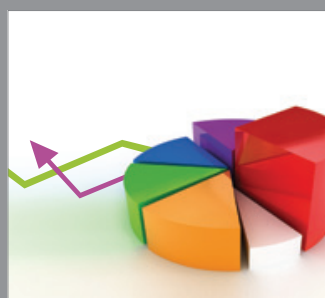

ournal of

Probability and Statistics

Promensencen
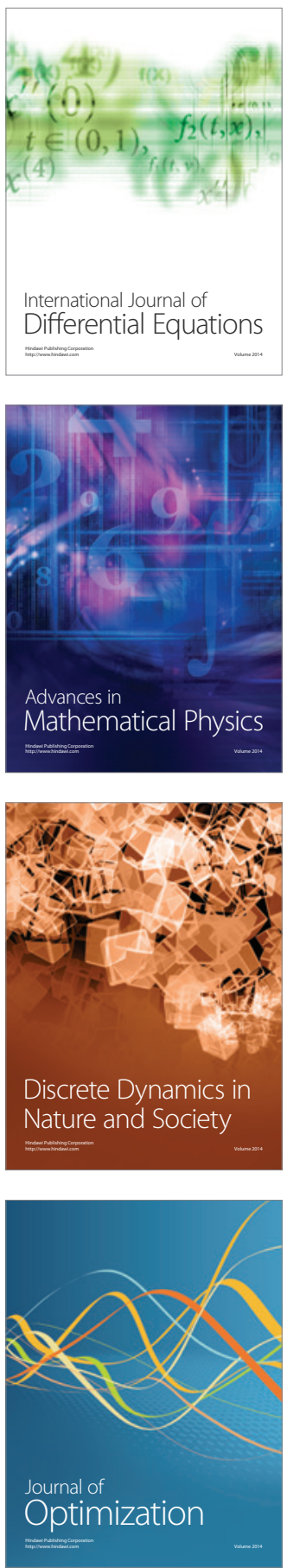